\title{
Nasopharyngeal Teratoma in a Neonate
}

\author{
Mohammad Reza Aramesha, Sahar Majidinezjad ${ }^{\mathrm{a}, \mathrm{c}}$, \\ Huda Ilkhanipak ${ }^{\mathrm{b}}$, Mehran Peyvasteh ${ }^{\mathrm{b}}$
}

\begin{abstract}
Teratomas are rare neoplasms in the nasopharyngeal area. They are composed of all germ cell layers and tend to be benign in all sites. Teratomas can cause dysphagia and respiratory problems. Diagnosing them antenatally helps to be prepared for possible complications that may occur after birth. Rised maternal AFP levels and polyhydramnios are seen and prompt further evaluations. In this case we present a Nasopharyngeal teratoma in a baby girl which occluded the airway and leaded to intubation. She developed seizures during her admission and since the mass was near to the CNS, MRI and neurological evaluations were preformed prior to the surgery. The mass was removed surgically after controlling the seizures with anticonvulsive therapy and ruling out any possible connections between the mass and the CNS. She was extubated two days after the surgery and was discharged after being successfully orally fed.
\end{abstract}

Keywords: Teratoma; Seizure; Nasopharynx

\section{Introduction}

Teratomas in the upper aerodigestive tract are rare neoplasms, accounting for fewer than $2 \%$ of all teratomas. Teratomas may be seen in the adult population but the majority occurs in the newborns or infants.

The most common location for teratomas within the aerodigestive tract mucosa is the nasopharynx, and other less commonly involved sites include the oral cavity (tonsils, tongue, palate), sinonasal cavity, the ear and the temporal bone. Nasopharyngeal teratomas present as a mass protruding in to the oral cavity or pharynx causing associated dysphagia and/or airway obstruction. Teratomas may be associated with maternal hydramnios and still birth [1].

Manuscript submitted February 18, 2020, accepted March 16, 2020

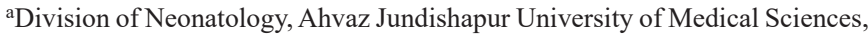
Ahvaz, Iran

${ }^{b}$ Division of Pediatric Surgery, Ahvaz Jundishapur University of Medical Sciences, Ahvaz, Iran

${ }^{\mathrm{c} C o r r e s p o n d i n g ~ A u t h o r: ~ S a h a r ~ M a j i d i n e z j a d, ~ D i v i s i o n ~ o f ~ N e o n a t o l o g y, ~ A h v a z ~}$ Jundishapur University of Medical Sciences, Ahvaz, Iran.

Email: Sahar.majidinezjad@gmail.com

doi: https://doi.org/10.14740/jnr578
Teratomas are composed of all three germ layers (ectodermal, mesodermal and endodermal). There is no gender predisposition. Histologically, teratomas are composed of mixed solid and cystic elements which may be mature, organized or immature. Neonatal teratomas from all sites tend to be benign [2]. In the nasopharyngeal area, they may appear in the midline or lateral pharyngeal wall and appear heterogeneous on magnetic resonance imaging (MRI) with fat and bone [3].

Persistently high maternal alpha-fetoprotein (AFP) level may be suggestive of teratoma and may be used to monitor for disease progression. Prenatal diagnosis with ultrasound and MRI, along with multidisciplinary prenatal care, is critical to evaluate the degree and extent of airway obstruction, and an EXIT procedure may be indicated in severe cases [4].

Here we present a case of nasopharyngeal teratoma in a neonate which is interesting because it was close to the central nervous system (CNS) and we had concerns about its connection to the brain and at the same time it caused breathing problems by occluding the airway.

\section{Case Report}

A baby girl was born on October 20, 2019, in Ahvaz, Iran, as a full term spontaneous delivery with a birth body weight of $3 \mathrm{~kg}$. The baby had respiratory distress and on physical examination, a red mass (approximately $3 \times 2 \times 1 \mathrm{~cm}$ ) was observed connected to the soft palate which occluded the larynx and caused respiratory distress (Fig. 1). There were no other abnormalities observed on physical examination. The baby was intubated and underwent mechanical ventilation and developed a right-sided pneumothorax. For further evaluation and surgical excision, she was referred to our center. On her chest X-ray, no pathological findings were observed and the chest tube was removed since the pneumothorax was totally resolved. We performed a spiral neck computed tomography (CT) scan which revealed a hypodense mass (with fat and soft tissue density) measuring $20 \times 15$ $\times 10 \mathrm{~mm}$ in the nasopharyngeal area, suggesting teratodermoid.

She had some abnormal movements which we considered seizures, we evaluated the electrolyte levels and the blood sugar and the results were normal. We could control those movements with intravenous phenobarbital $(10 \mathrm{mg} / \mathrm{kg}$ loading dose and 5 $\mathrm{mg} / \mathrm{kg} /$ day maintenance) and they were not repeated after anticonvulsant therapy. In order to rule out any connections to the brain, we performed a brain MRI and neurosurgical consult, and there was no connection between the mass and the brain (Fig. 2). On her echocardiography, no structural cardiac anomalies were reported. 


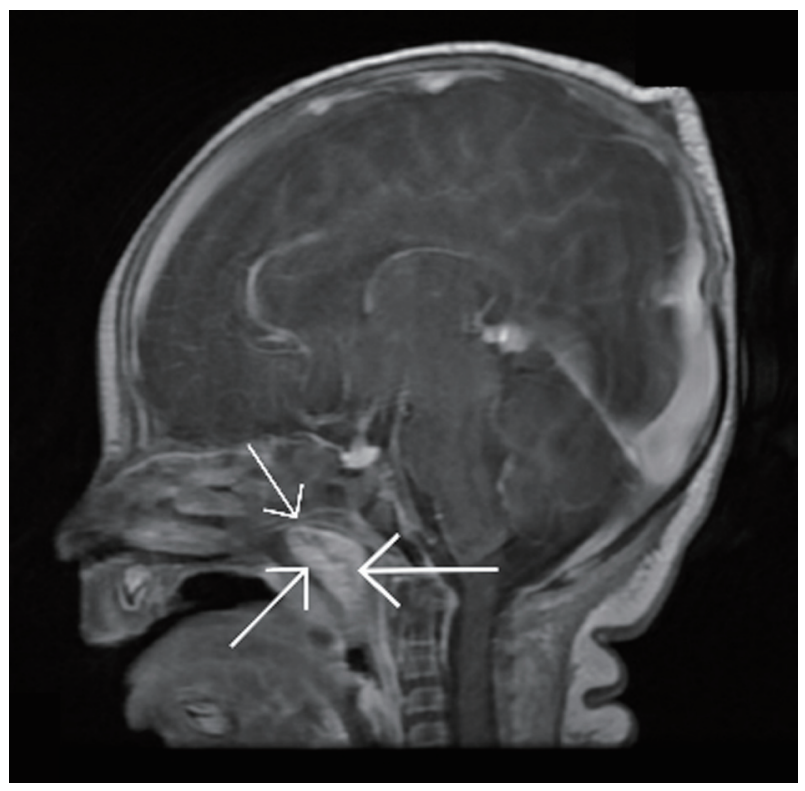

Figure 1. Brain MRI (sagittal view): the arrows show the mass in the nasopharyngeal area occluding the airway.

She was prepared for surgery and underwent surgery 2 days after admission to our center. Intraoperatively a mass arising from the nasopharyngeal area was observed and covered the larynx and the vocal cords. Access to the oral cavity was maintained with retractors applied over the upper and lower jaws (Fig. 3). The mass was pulled aside with sutures while the airway was

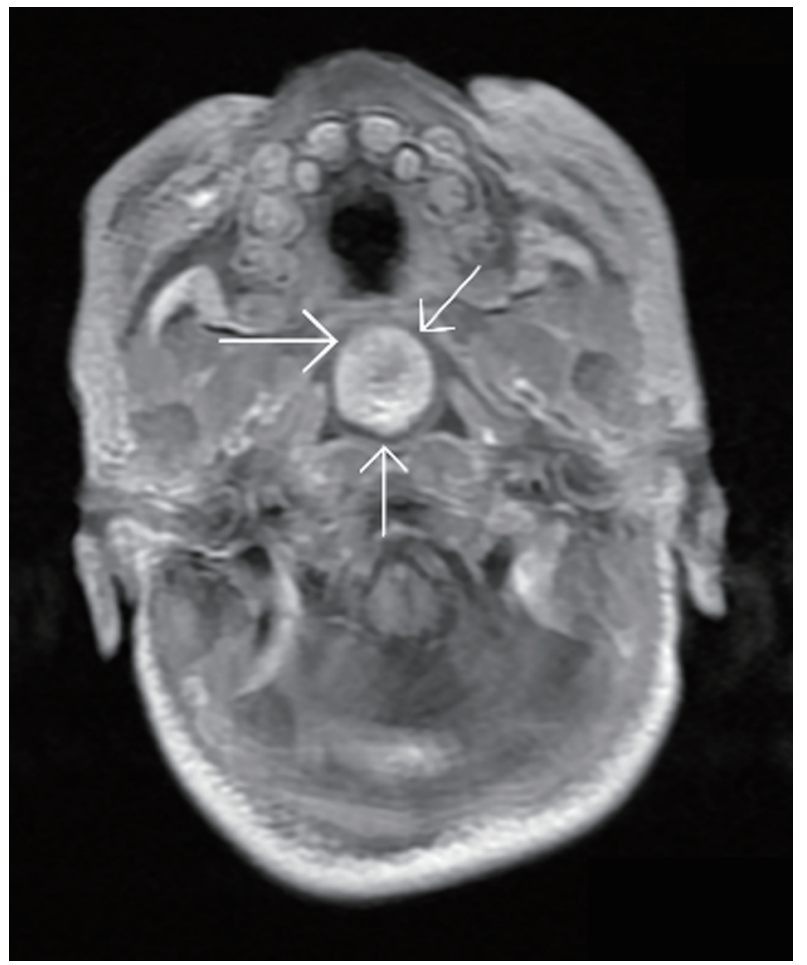

Figure 2. Brain MRI (axial view): there is no connection between the mass and the CNS (the arrows show the mass).

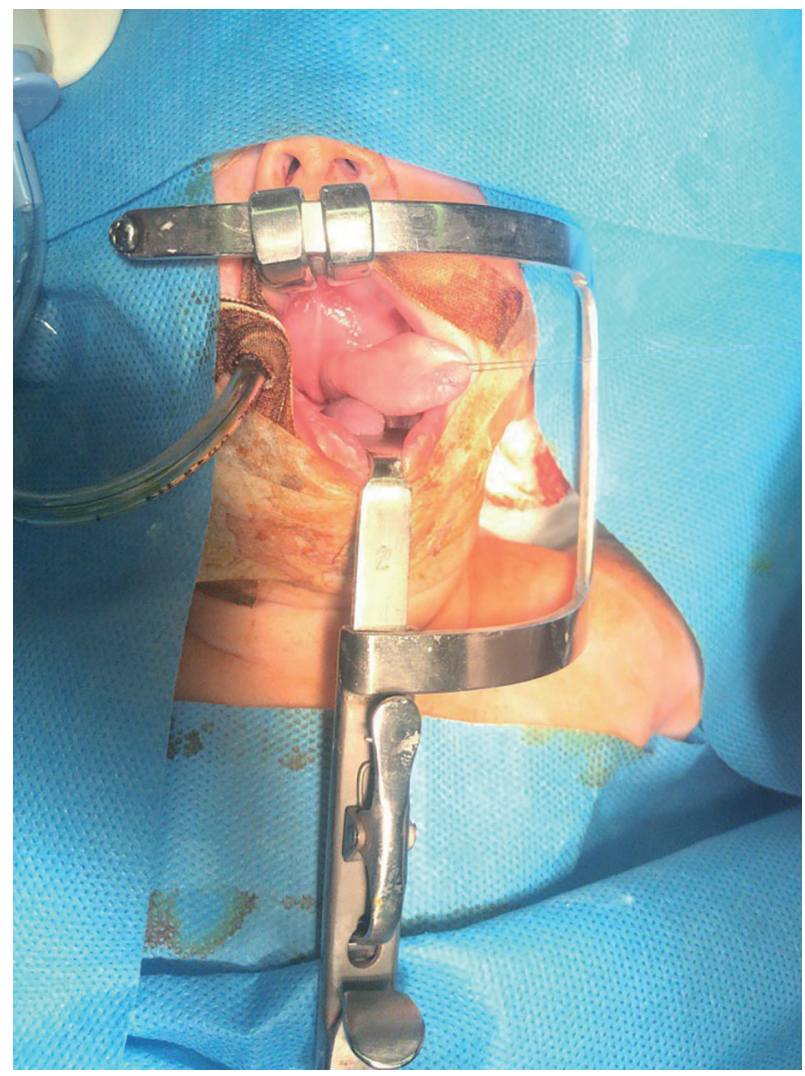

Figure 3. The mass is pulled aside and the airway is protected during surgery.

protected. The mass was excised with a few mms of healthy margins (Figs. 4 and 5). Bleeding and manipulation of the airway were minimal. After the surgery, the patient was transferred to the neonatal intensive care unit (NICU) and she was extubated after 2 days. Finally she was discharged after being successfully orally fed. Histopathological examination showed a tumor without malignancy and invasion to the margins, and it was compatible with mature cystic teratoma (dermoid cyst). Since her seizures were controlled with phenobarbital, we tapered the dose to $3 \mathrm{mg} / \mathrm{kg}$ and discharged her with oral phenobarbital and recommended close follow-up before discontinuing the drug.

\section{Discussion}

Teratomas occur in 1 out of 4,000 live births. Head and neck teratomas are generally localized in the neck and nasopharyngeal area and comprise $1-10 \%$ of cases [5].

Teratomas in childhood are commonly found in the sacrococcygeal region, the gonads and the mediastinum, whereas these lesions are distinctly rare in the head and neck [6].

Teratomas have four main subgroups: 1) Dermoids, which contain tissues of mesoderm and epidermal origin; 2) Treaties, which contain tissue from three primary germ layers but are poorly differentiated; 3 ) True teratomas, which are similar to teratoids but differentiated into recognizable tissues; and 4) Epiganthi, which are also tridermal in origin, but differentiated 


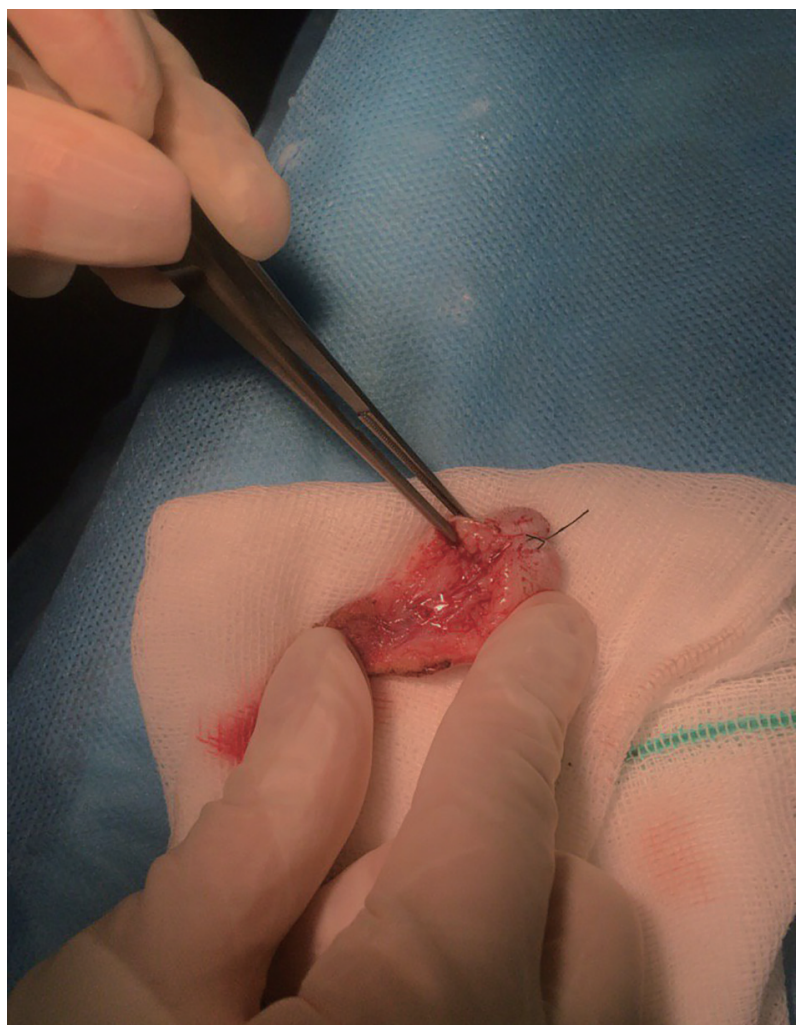

Figure 4. The excised mass; further pathological study revealed it was teratodermoid.

into recognizable organs, sometimes with limbs or even a second visible fetus. Our case was a teratodermoid [6].

Teratomas are commonly diagnosed antenatally on ultrasonography, with larger ones usually associated with maternal polyhydramnios because it interferes with fetal swallowing [6]. Since nasopharyngeal teratomas can block the airway for safer and better management, antenatal diagnosis is advocated. In our case blockage of the airway was the most concerning problem. The mass effect of a teratoma in the nasopharyngeal area can lead to difficult intubation and subsequent hypoxia. Therefore, a multidisciplinary prenatal care and expert physicians familiar with problematic neonatal intubations present at the time of birth are critical to avoid any complications.

Preoperative CT scan evaluation can be helpful for defining the tumor's origin, determining the anatomical extent and possible connections of the tumor.

The aim of surgical management was to remove the mass with healthy margins and provide a secure airway. It is better to perform the surgery soon after stabilizing the baby because prolonged intubation and ventilator support impose risks on the neonate.

It is important to diagnose such cases antenatally, and refer the mother to a tertiary center for delivery to avoid possible complications in management and transfer. This baby was not born in our center and possible complications of transferring an intubated baby could be avoided if her mother was referred to our center before giving birth.

In this case the mass was close to the CNS and the baby

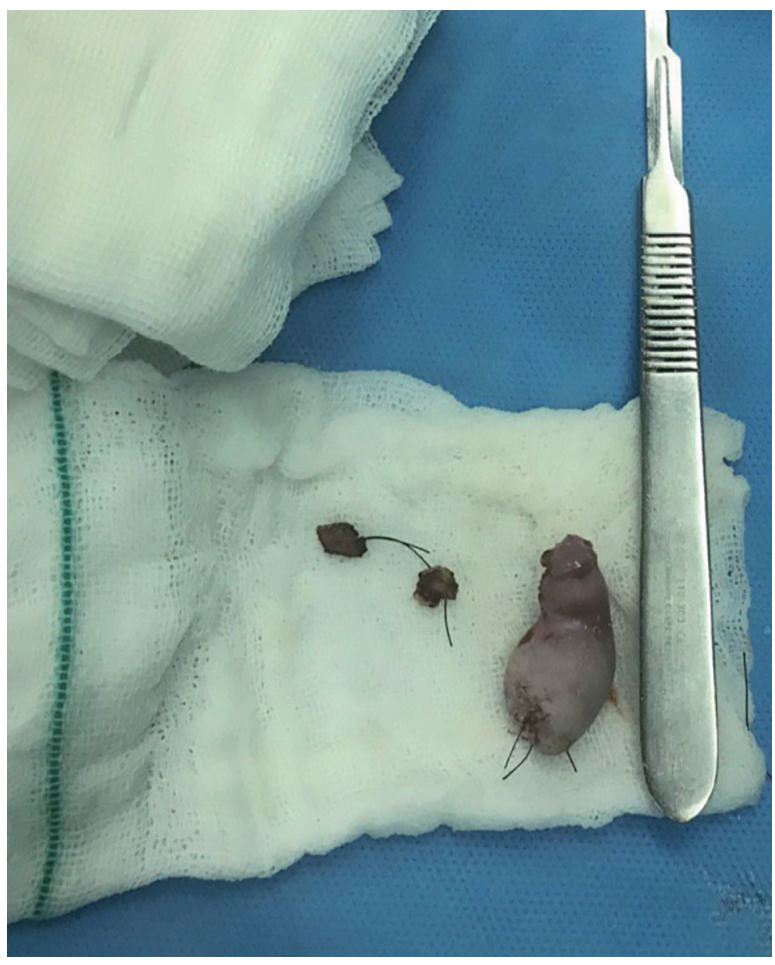

Figure 5. The excised mass, measuring $30 \times 20 \times 10 \mathrm{~mm}$.

had seizures which were controlled with phenobarbital. It seems reasonable to rule out any possible connections between such masses and the CNS especially when we have neurological symptoms and the mass is close to the CNS, so we did an MRI and neurosurgical consult and it was confirmed that there is no connection between the mass and the CNS and the mass was excised without any complications.

In similar cases we recommend antenatal diagnosis, referral to a tertiary center for delivery, evaluation of possible connections prior to the surgery with CT and/or MRI.

\section{Acknowledgments}

None to declare.

\section{Financial Disclosure}

None to declare.

\section{Conflict of Interest}

None to declare.

\section{Informed Consent}

Informed consent was obtained. 


\section{Author Contributions}

All authors contributed in the literature search and writing the manuscript equally.

\section{Data Availability}

The data supporting the findings of this study are available from the corresponding author upon reasonable request.

\section{References}

1. Wenig B. Tumors of the upper respiratory tract. In: Fletcher C. Diagnostic Histopathology of the Tumors, volume 1, 4th edition. Saunders, Philadelphia. 2013:110.

2. Brandwein MS. Hairy polyps and teratomas. In: Brandwein MS: Textbook of Head and Neck Pathology, 1st volume, 1st edition. Springer, Switzerland. 2016:134.

3. Ottenson T, Wang T. Upper airway lesions in the neonate. In: Martin RJ, Fanaroff AA, Walsh MC. Neonatal-Perinatal medicine. Volume 2, 11 th edition. Elsevier, Philadelphia. 2020:1247.

4. Paradis J, Koltai PJ. Pediatric teratoma and dermoid cysts. Otolaryngol Clin North Am. 2015;48(1):121-136.

5. Mirshemirani A, Khaleghnejad A, Mohajerzadeh L, Samsami M, Hasas-Yeganeh S. Congenital nasopharyngeal teratoma in a neonate. Iran J Pediatr. 2011;21(2):249252.

6. Hassan S, Sidek DS, Shah Jihan WD, Phutane G, Mutum SS. Massive lingual teratoma in a neonate. Singapore Med J. 2007;48(8):e212-214. 